\title{
CHARACTERIZATION OF VINE CANOPY THROUGH TWO DIMENSIONAL IMAGING
}

\author{
Francesco Marinello ${ }^{1,2}$, Giulio Toniolo ${ }^{1}$, Tatevik Yezekian ${ }^{3}$, Luigi Sartori ${ }^{1,2}$ \\ ${ }^{1}$ TeSAF Department, University of Padova, Italy; \\ ${ }^{2}$ NEOS srl, Spin off of the University of Padova, Italy; \\ ${ }^{3}$ Institute of Technology, Estonian University of Life Sciences, Estonia \\ francesco.marinello@unipd.it
}

\begin{abstract}
In the last decade, reconstruction of plant dimensions and volume has gained a noticeable importance, in particular for the possibility of collecting data correlated to biomass, leaves area, etc. This is specifically of interest in the case of vineyards, where knowledge of variability can be not only a useful mean to evaluate the health condition of the vines and of the grapes, but also an important input to allow variable management practices. Different sensing technologies are available for shape reconstruction, as for instance ultrasonic sensors, laser scanners or depth cameras, however, their practical application is still limited by low quality information, high costs, or high speed data processing demand. For the present work two-dimensional imaging is proposed as a viable solution for shape reconstruction of canopies. The method takes advantage of low cost $2 \mathrm{D}$ commercial cameras, which can be installed on board the tractor allowing on the go collection of images of plants from the bottom. The paper describes the instrumentation set up and integration with a specific thresholding algorithm allowing segmentation of canopy profile. Tests were carried out on 20 different dates on a glera vineyard in an experimental farm in the North of Italy. The results were correlated with the number of leaves, leaf area index and canopy volume. High correlation was identified in the case of volume with coefficients of determination $R^{2}>0.7$ in most of the cases.
\end{abstract}

Keywords: canopy, profile, imaging, segmentation.

\section{Introduction}

The opportunity of analyzing the variable profile of vineyard canopies is of the highest relevance for the possibility of precisely quantifying local biomass. Such interest arises from the correlation, which is typically found between the canopy size and plant needs in terms of water and nutrients as well as with the yield and quality [1]. As a consequence, knowledge of plant dimensions allows not only evaluation of the plant health conditions, but also exploitation of variable rate and management practices, including crop protection and canopy management [1-3].

Many researches and approaches have been proposed in the last decade, which allow quantification of the plant dimensions $[1 ; 3 ; 4]$, also taking advantage of the availability of miniaturized low cost sensors and of an increased speed in data processing [5;6]. Available technologies can be referred mainly to ultrasonics, laser sensors and other optical methods [7].

Ultrasonic technology is a low cost solution, which relies on the sound waves echoing off of a given target $[8 ; 9]$ to provide its external contour. Its performance is influenced by ultrasound propagation, which often exhibits parasitic signals or losses especially in the cases of thin targets or high reflection angles, as is in the case of small branches or in the case of badly oriented leaves. Additionally, ultrasonic sensors are very much sensitive to humidity, dust and high temperatures, however, their practical implementation has provided interesting results especially coupled with some recent spraying machines, to allow variable rate distributions.

Laser based techniques include an interesting group of sensors, which take advantage of coherent light sources shined into the scene. The three dimensional reconstruction is achieved by implementing either a triangulation or a time of flight approach, and the effectiveness of the method has been already demonstrated in a number of applications, including vineyards [4; 10]. Main limitations are ascribable to distortions and noise mainly caused by sunlight exposure and to high computational complexity.

Other optical methods are based on two-dimensional RGB images taken with standard CCD (Charge-Coupled Device) or CMOS (Complementary Metal-Oxide-Semiconductor) cameras. In the so called structure from motion approach, images captured from adjacent positions are collected and processed applying well known principles of photogrammetry to provide three-dimensional profiling. Other approaches use spectral information to allow canopy identification and segmentation [11]. In both cases limitations are still to be ascribed to the need for robust algorithms and powerful processors, especially when high resolution imaging is needed. Additionally, some attempt has been recently proposed to use digital imaging to characterize the dimensions of vine sticks [12], however its 
practical application is limited by the need to position a white contrasting panel behind the vines and a red reference mark on the wires.

The present work introduces a new optical approach, which relies on implementation of low cost RGB sensors. In fact, the cost of such sensors can be as low as 2-3 EUR per Mpixel, and low processing capacity is needed. Canopy profile is extracted taking advantage of the specific positioning of the camera and of implementation of a robust segmentation algorithm. The sensor was statically positioned into a vineyard and implemented to collect canopy profiles from vine plants (Figure 1-1). A schematic representation of a possible application of the new proposed approach is shown in Figure 12 , where the RGB sensor is mounted on an agricultural tractor and a feedback is generated to allow regulation of the agricultural implement.
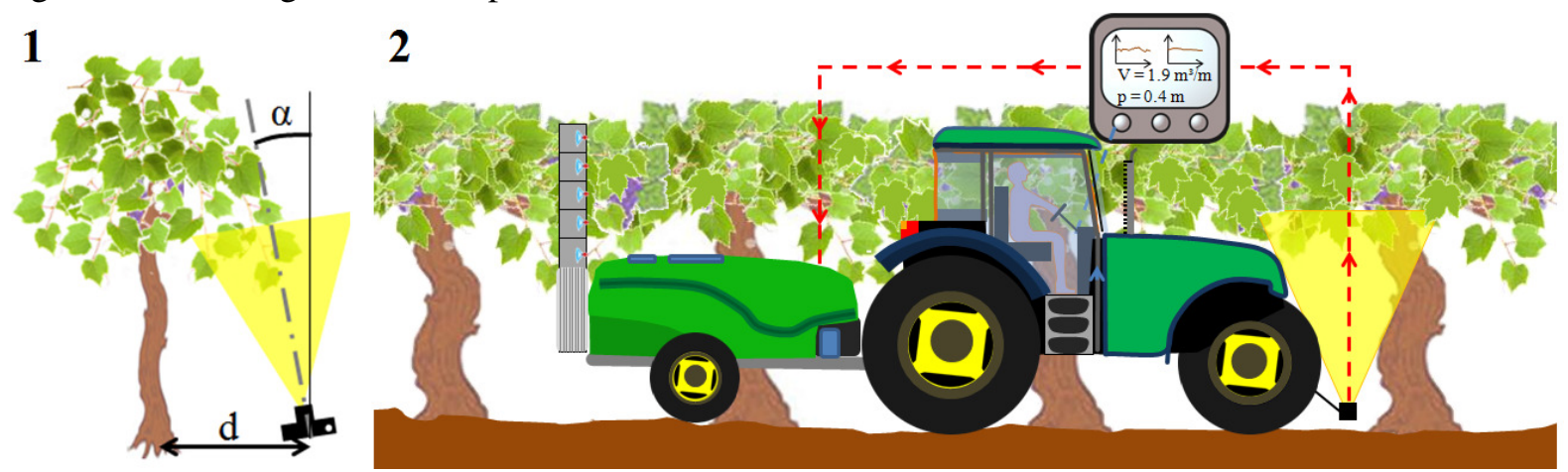

Fig. 1. Schematic representation of the new monitoring method: 1 - RGB sensor scanning the canopy from the bottom; 2 - possible solution with the sensor installed in front of a tractor to allow variable rate distribution with an attached implement

\section{Materials and methods}

For the present work, a commercial RGB color sensor with a standard infrared filter was implemented. The sensor is a CMOS type, with a $23.2 \times 15.4 \mathrm{~mm}$ size and $6016 \times 4000$ pixels resolution and a 12 bit depth. Images were collected from the bottom, as depicted in Figure 1-1. Such particular layout was deliberately chosen in order to help subsequent image processing operations. Indeed, the specific scan from the bottom set up produces images which feature mainly canopy and sky portions. Such partitioning is variable and depends upon specific position of the camera and canopy volume. Therefore, during the experiments, different conditions were tested, with the camera placed at a distance $d$ ranging between 0.4 and $0.7 \mathrm{~m}$ and a relative angle $\alpha$ ranging between $45^{\circ}$ and $90^{\circ}$.

Two examples of captured images are reported in the first column of Figures 2 and 3. In the two cases, different meteorological conditions are recognizable: scattered clouds and clear sky respectively in the first and in the second. In both cases the different distance between canopy and sky and the different degree of illumination produce an image where canopy and non canopy areas are clearly identifiable. It is worth noting how such a clear difference would not be possible in the case of scans from the top or scan from aside. Indeed, in the first case the definition of canopy lateral profile would be confused by the presence of inter-row grass cover, while in the second case the canopy lateral profile can be appreciated only taking advantage of three-dimensional techniques [3; 4].

The developed method relies on a robust algorithm, which can be summarized as follows:

- color intensity distribution histograms are produced for the analyzed image (Figure 2-2);

- average curve is obtained from the three color channels: red, green and blue (Figure 2-3);

- center of mass $c$ of the higher intensity peak is calculated (Figure 2-4);

- image is thresholded simply applying a band stop filter with a cut-off intensity equal to the detected intensity $c$ and a variable width $T$ ranging between $10 \%$ and $100 \%$ of the total color width, with $10 \%$ steps (Figures 2-5 and 3);

- after thresholding, the image is binarized in order to allow extraction of the canopy profile, which is eventually used to estimate canopy projected area $A$ (Figure 2-6).

The filtering operation produces a segmented image, where the canopy is progressively isolated from the rest of the sky as can be more clearly noticed in Figure 3. 

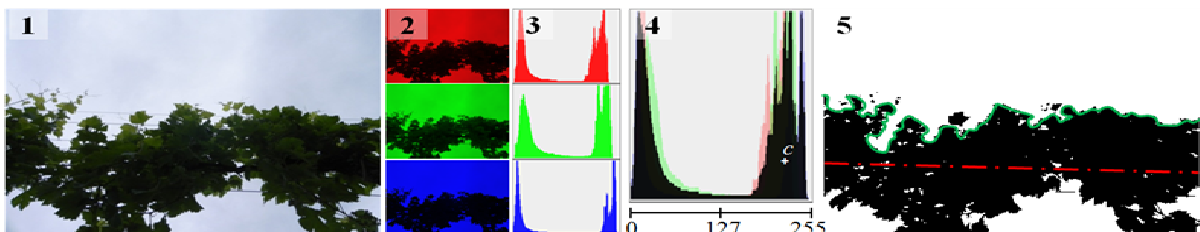

Fig. 2. Step by step representation of the proposed method: 1 - image collection; 2 - separation of red, green and blue channels; 3 - quantification of intensity distribution histograms; 4 - definition of the center of mass $c ; 5$ - thresholding; 6 - extraction of canopy projected area $A$

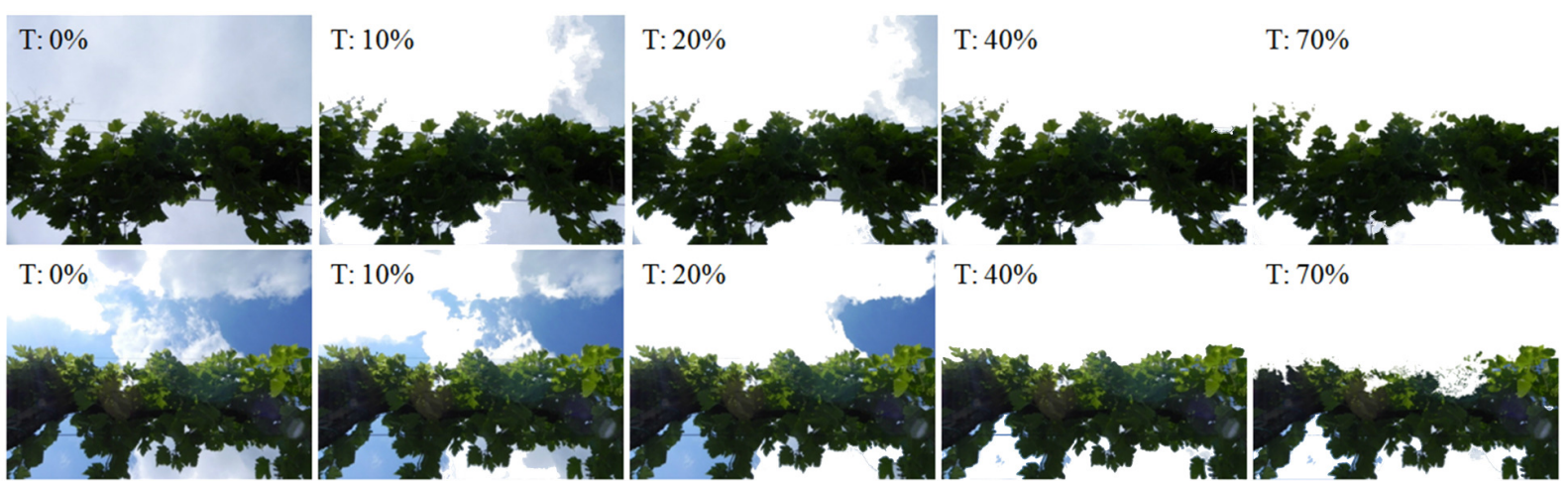

Fig. 3. Two collected vines images post-processed at different threshold levels $T$

It should be noted that relatively high filter widths $T$ increase the effectiveness in filtering out sky portions from the image, but at the same time increase the risk of filtering out canopy portions. Therefore, the robustness of the algorithm depends on the ability of defining the filtering width providing the best trade off. This can be achieved after identification of the plateau region in the segmentation process. As can be noticed in Figure 4, where the segmented area $A$ is reported as a function of the filter width $T$ for 50 different canopy images, a repeatable shape can be identified, schematically represented by the red dotted line. Repeatable and robust identification of proper filtering width for a given image was therefore based on the position of the inflection point for the curve associated to the same image.

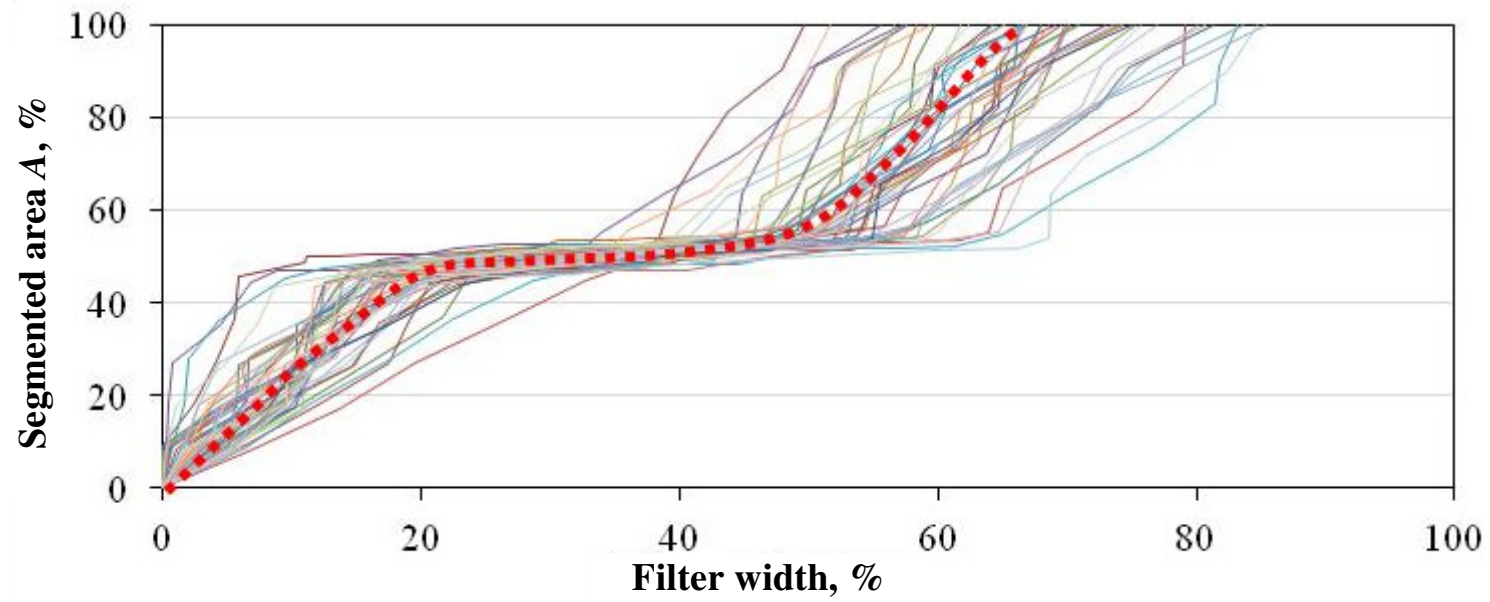

Fig. 4. Segmented area as a function of filter width: curves refer to 50 different images taken on different vineyard sections, under different meteorological conditions

The experimental application of the method was repeated in two different periods:

- the first during the vegetative growth from late April until the beginning of June, when the first leaf trimming operations are carried out

- the second after harvesting, between October and November, when the canopy volume is much smaller. 
The study was carried out in a private farm in north-eastern Italy in a typical Po Valley vineyard $(45.91 \mathrm{~N}, 12.34 \mathrm{E})$. Glera grapevine variety was available for the tests, managed through a Sylvoz pruning system. Grape branches typically lay at a $1.2-1.3 \mathrm{~m}$ height. Vine-stocks were planted with a $2.8 \mathrm{~m}$ inter-row and $1.3 \mathrm{~m}$ intra-row spacing (corresponding to 2750 plants per hectare). The vineyard is east-west oriented, thus receiving an optimal sun radiation during daylight. However, it is worth noting that sun radiation is not affecting the measurement procedure and conversely is of help for an effective characterization of vines.

A total of 500 images were captured from two different combinations of distance and relative inclination: in the first case the camera was positioned at a relative distance $d=0.4 \mathrm{~m}$ from the plant and parallel to the soil $\left(\alpha=90^{\circ}\right)$, while in the second case the distance was increased to $d=0.7 \mathrm{~m}$ and the inclination reduced to $\alpha=45^{\circ}$. The images were processed taking advantage of the proposed algorithm. The collected data were correlated with the results arising from manual measurements on volume and leaf area, carried out on the same vine portions collected through the new proposed optical approach. Manual measurements of the volume and leaf area were done applying the same procedure described in [4]. For the volume, widths at three different heights were measured considering as a reference surface the virtually flat plane passing through the trellising supporting the plants. The three widths were measured on parallel positions at a relative distance of about $33 \mathrm{~cm}$, in order to have 4 partial volume values over $1 \mathrm{~m}$ length. A total of 3000 manual measurements were then collected. For the leaf area, the procedure included counting the number of leaves per vine and estimation of the area of the leaves through implementation of a digital camera and a reference grid [13]. A total of 2500 leaves was eventually considered.

\section{Results and discussion}

The proposed technique has been developed in order to allow fast extraction of information related to vine canopy. Specifically it is assumed that scan from the bottom can provide quantification of canopy volume and of leaf area. To support such assumption, the extracted canopy area was studied in correlation with leaf area and volume parameters. The main results are reported in Figure 4: it can be noticed how a good linearity is obtained particularly in the case of canopy volume. Only at higher growing stages, a deviation from linearity is recognizable. Such deviation is most probably due to an overestimation of the projected area, occurring especially when the bottom of the canopy, which is closer to the camera, grows occupying an abnormally larger portion of the field of view. Due to this effect, a second degree polynomial model is preferable, as shown in Figure 4-1. The deviation is less evident when the sensor is positioned at a higher distance from the plant $\left(d=0.7 \mathrm{~m} ; \alpha=45^{\circ}\right)$, and the bottom canopy branches are more distant from the camera.
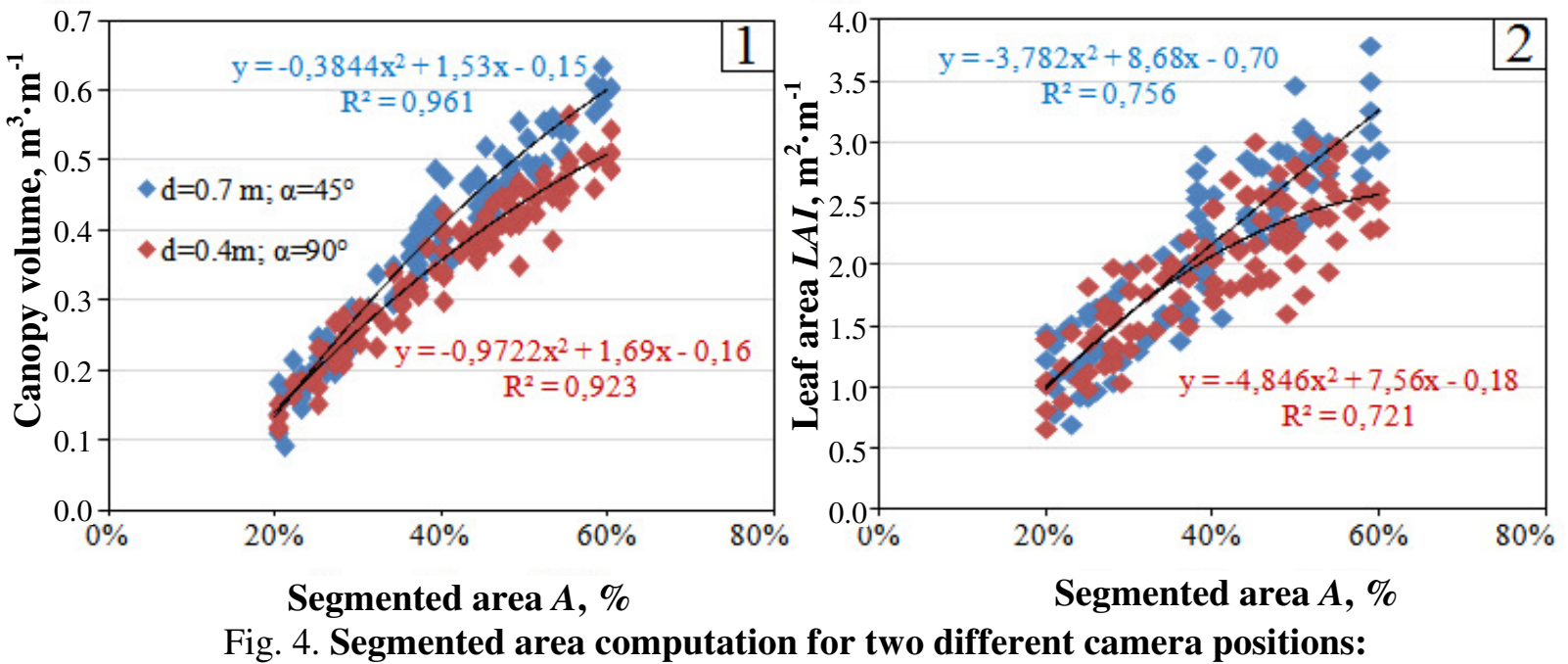

Fig. 4. Segmented area computation for two different camera positions:

1 - correlated to canopy volume and 2 - correlated to leaf area

As a result, the best fitting polynomial curves exhibit a lower second degree coefficient in the case of the higher distance camera. In both cases a high correlation is found, as demonstrated by high coefficients of determination $\left(R^{2}=0.961\right.$ and $R^{2}=0.923$ respectively for $d=0.7$ and $\left.0.4 \mathrm{~m}\right)$. In both 
cases a standard error $S E=0.03 \mathrm{~m}^{3} \cdot \mathrm{m}^{-1}$ was estimated, corresponding to the $5-30 \%$ of the measured canopy volumes. Particularly in the case of larger canopies, when the volumes exceed $0.3 \mathrm{~m}^{3} \cdot \mathrm{m}^{-1}$ (from May onwards in the studied case), the proposed method allows characterization of the plant dimensions with a contribution of uncertainty which is lower than $10 \%$. Such value can be considered to be acceptable for exploitation of variable rate and management practices.

If, on the one hand, the volume of canopy is helpful to determine the growth of the plant, on the other hand, the vineyard management can benefit from information on the overall area of the leaves. Accordingly, the leaf area index (LAI) is one of the most used parameters in viticulture related to the foliage $[3 ; 14 ; 15]$. For this reason the collected values on the segmented area $A$ were correlated also to the data arising from the leaf area analysis. The main results are reported in Figure 4-2, again with values sorted for the two different camera positions. As for the volume, a slight deviation from linearity can be noticed at higher canopy dimensions, more evident for the camera positioned in a closer position with respect to the vines. However, for both camera positions the second degree polynomial curve presents an acceptable degree of correlation as demonstrated by the relatively high coefficients of determination $\left(R^{2}=0.756\right.$ and $R^{2}=0.721$ respectively for $d=0.7$ and $\left.0.4 \mathrm{~m}\right)$. In the two cases a standard error ranging between 0.30 and $0.33 \mathrm{~m}^{2} \cdot \mathrm{m}^{-1}$ was estimated, corresponding to the 10$30 \%$ of the measured leaf areas. Such contribution of uncertainty is clearly higher than in the case of the volume estimation, nevertheless, it can be considered to be acceptably low from May onwards, when the average leaf area exceeds $2.0-2.5 \mathrm{~m}^{2} \cdot \mathrm{m}^{-1}$. It should be noted that both in the case of volume and area calculation, quantitative data can be affected by deviations of the tractor from a linear trajectory and by vehicle vibrations and roll oscillations, however, implementation of automatic guidance systems and self balanced pivoted support can minimize such distortions.

\section{Conclusions}

1. The present work proposes implementation of a standard RGB camera as a cost effective solution for two-dimensional characterization of vine canopy profiles.

2. CMOS or CCD sensors, which can be applied for the canopy profile extraction, typically cost less than 10 EUR, and low post processing and computing capacities are needed.

3. The experiments have shown a high correlation between the digital analysis and hand measurement results on the canopy volume and to some extent on the leaf area.

4. The new approach is less effective in vine early stages when the leaves are too small, thus negatively affecting the definition of the canopy profile.

5. The method has demonstrated to be effective under different meteorological conditions, but exploits higher performances in case of medium to high sun radiation.

6. The tests have been performed on Glera, but the measuring approach can be easily extended to different grapevines varieties.

\section{Aknowledgement}

The study is supported by a grant from the University of Padova (code CPDA143174), Italy. We also gratefully acknowledge the support of NVIDIA Corporation with the donation of the Tesla K40 GPU used within the present research.

\section{References}

1. Escolà A., Martínez-Casasnovas J.A., Rufat J., Arnó J., Arbonés A., Sebé F., Pascual M., Gregorio E., Rosell-Polo J.R., Mobile terrestrial laser scanner applications in precision fruticulture/horticulture and tools to extract information from canopy point clouds. Precision Agriculture, vol. 18/1, 2017, pp. 111-132.

2. Basso B., Dumont B., Cammarano D., Pezzuolo A., Marinello F., Sartori L., Environmental and economic benefits of variable rate nitrogen fertilization in a nitrate vulnerable zone. Science of the Total Environment, vol. 545-546, 2016, pp. 227-235.

3. Arnó J., Escolà A., Vallès J.M., Llorens J., Sanz R., Masip J., Palacín J., Rosell-Polo J.R., Leaf area index estimation in vineyards using a ground-based LiDAR scanner. Precision Agriculture, vol. 14/3, 2013, pp. 290-306. 
4. Marinello F., Pezzuolo A., Meggio F., Martínez-Casasnovas J.A., Yezekyan T., Sartori L., Application of the Kinect sensor for three dimensional characterization of vine canopy. Advances in Animal Biosciences: Precision Agriculture (ECPA) 2017, vol. 8/2, 2017.

5. Marinello F., Schiavuta P., Cavalli R., Pezzuolo A., Carmignato S., Savio E., Critical factors in cantilever near-field scanning optical microscopy. IEEE Sensors Journal, vol. 14/9, 2014, pp. 3236-3244.

6. Pajares G., Peruzzi A., Gonzalez-de-Santos P. Sensors in Agriculture and Forestry. Sensor, vol. 13, 2013, pp. 12132-12139.

7. Su X., Zhang Q., Dynamic 3-D shape measurement method: A review. Optics and Lasers in Engineering, vol. 48/2, 2010, pp. 191-204.

8. Palleja T., Landers A.J., Real time canopy density validation using ultrasonic envelope signals and point quadrat analysis. Computers and Electronics in Agriculture, vol. 134, 2017, pp. 43-50.

9. Tang J., Woods M., Cossell S., Liu S., Whitty M., Non-Productive Vine Canopy Estimation through Proximal and Remote Sensing. IFAC-PapersOnLine, vol. 49/16, 2016, pp. 398-403.

10. Marinello F., Pezzuolo A., Gasparini F., Arvidsson J., Sartori L., Application of the Kinect sensor for dynamic soil surface characterization. Precision Agriculture, vol. 5, 2015, pp. 1-12.

11. Dubbini M., Pezzuolo A., De Giglio M., Gattelli M., Curzio L., Covi D., Yezekyan T., Marinello F., Last generation instrument for agriculture multispectral data collection. CIGR Journal, 2017.

12. Oliveira P.C., Moura J.P., Fernandes L.F., Amaral E.M., Oliveira A.A., A non-destructive method based on digital image processing for calculate the vigor and the vegetative expression of vines. Computers and Electronics in Agriculture, vol. 124, 2016, pp. 289-294.

13. Fila G., Gardiman M., Belvini P., Meggio F., Pitacco A., A comparison of different modelling solutions for studying grapevine phenology under present and future climate scenarios. Agricultural and Forest Meteorology, vol. 195-196, 2014, pp. 192-205.

14. Zorer R., Rocchini D., Metz M., Delucchi L., Zottele F., Meggio F., Neteler M., Daily MODIS land surface temperature data for the analysis of the heat requirements of grapevine varieties. IEEE Transactions on Geoscience and Remote Sensing, vol. 51/7, 2013, pp. 2128-2135.

15. Corso M., Vannozzi A., Maza E., Vitulo N., Meggio F., Bouzayen M., Valle G., Bonghi C., Lucchin M., Transcriptome pathways in leaf and root of grapevine genotypes with contrasting drought tolerance. Acta Horticulturae, vol 1136, 2016, pp. 161-168. 